\title{
On the rod placement theorem of Rybko and Shlosman ${ }^{1}$
}

\author{
Venkat Anantharam ${ }^{2}$ \\ EECS Department \\ University of California \\ Berkeley, CA 94720, U.S.A. \\ ananth@eecs.berkeley.edu \\ $1-510-643-8435(\mathrm{O})$ \\ $1-510-642-2845$ (fax)
}

Short title: Rod placement theorem

Keywords: FCFS queue, FIFO queue, rod placement, symmetric group.

\begin{abstract}
Given $n-1$ points $x_{1} \leq x_{2} \leq \ldots \leq x_{n-1}$ on the real line and a set of $n$ rods of strictly positive lengths $\lambda_{1} \leq \lambda_{2} \leq \ldots \leq \lambda_{n}$, we get to choose an $n$-th point $x_{n}$ anywhere on the real line and to assign the rods to the points according to an arbitrary permutation $\pi$. The $\operatorname{rod} \lambda_{\pi(k)}$ is thought of as the workload brought in by a customer arriving at time $x_{k}$ into a first in -first out queue which starts empty at $-\infty$. If any $x_{i}$ equals $x_{j}$ for $i<j$, service is provided to the rod assigned to $x_{i}$ before the rod assigned to $x_{j}$.

Let $Y_{\pi}\left(x_{n}\right)$ denote the set of departure times of the customers (rods). Let $N_{\pi}\left(x_{1}, \ldots, x_{n-1} ; \lambda_{1}, \ldots, \lambda_{n}\right)$ denote the number of choices for the location of $x_{n}$ for which $0 \in Y_{\pi}\left(x_{n}\right)$. Rybko and Shlosman proved that

$$
\sum_{\pi} N_{\pi}\left(x_{1}, \ldots, x_{n-1} ; \lambda_{1}, \ldots, \lambda_{n}\right)=n !
$$

for Lebesgue almost all $\left(x_{1}, \ldots, x_{n-1} ; \lambda_{1}, \ldots, \lambda_{n}\right)$.

Let $y_{\pi, k}\left(x_{n}\right)$ denote the departure point of the rod $\lambda_{k}$. Let $N_{\pi, k}(y)$ denote the number of choices for the location of $x_{n}$ for which $y_{\pi, k}\left(x_{n}\right)=$ $y$ and let $N_{k}(y)=\sum_{\pi} N_{\pi, k}(y)$. In this paper we prove that for every $\left(x_{1}, \ldots, x_{n-1} ; \lambda_{1}, \ldots, \lambda_{n}\right)$ and every $k$ we have $N_{k}(y)=(n-1)$ ! for all but finitely many $y$. This implies (and strengthens) the rod placement theorem of Rybko and Shlosman.

\footnotetext{
${ }^{1}$ Version of 17 May 2005

${ }^{2}$ Research supported by ONR MURI N00014-1-0637, NSF ECS-0123512, Marvell Semiconductor, and the University of California MICRO program.
} 


\section{Discussion}

In this paper we prove a result which implies (and strengthens) the rod placement theorem of Rybko and Shlosman ([2]). For the basic facts we use from the theory of queues see, for instance, Asmussen ([1]).

\section{Theorem :}

Given $n-1$ points $x_{1} \leq x_{2} \leq \ldots \leq x_{n-1}$ on the real line and a set of $n$ rods of strictly positive lengths $\lambda_{1} \leq \lambda_{2} \leq \ldots \leq \lambda_{n}$, we get to choose an $n$-th point $x_{n}$ anywhere on the real line and to assign the rods to the points according to an arbitrary permutation $\pi$. The $\operatorname{rod} \lambda_{\pi(k)}$ is thought of as the workload brought in by a customer arriving at time $x_{k}$ into a first in -first out (FIFO) queue which starts empty at $-\infty$. If any $x_{i}$ equals $x_{j}$ for $i<j$, service is provided to the rod assigned to $x_{i}$ before the rod assigned to $x_{j}$.

Let $y_{\pi, k}\left(x_{n}\right)$ denote the departure point of the $\operatorname{rod} \lambda_{k}$. Let $N_{\pi, k}(y)$ denote the number of choices for the location of $x_{n}$ for which $y_{\pi, k}\left(x_{n}\right)=y$ and let $N_{k}(y)=\sum_{\pi} N_{\pi, k}(y)$. Then for every $\left(x_{1}, \ldots, x_{n-1} ; \lambda_{1}, \ldots, \lambda_{n}\right)$ and every $k$ we have $N_{k}(y)=(n-1)$ ! for all but finitely many $y$.

Remark :

Let $N_{\pi}\left(x_{1}, \ldots, x_{n-1} ; \lambda_{1}, \ldots, \lambda_{n}\right)$ denote the number of choices for the location of $x_{n}$ for which $0 \in Y_{\pi}\left(x_{n}\right)=\left\{y_{\pi, 1}\left(x_{n}\right), \ldots, y_{\pi, n}\left(x_{n}\right)\right\}$. Rybko and Shlosman $([2])$ proved that

$$
\sum_{\pi} N_{\pi}\left(x_{1}, \ldots, x_{n-1} ; \lambda_{1}, \ldots, \lambda_{n}\right)=n !
$$

for Lebesgue almost all $\left(x_{1}, \ldots, x_{n-1}, \lambda_{1}, \ldots, \lambda_{n}\right)$. To derive this from the theorem, since $0 \notin B$ for Lebesgue almost all $\left(x_{1}, \ldots, x_{n-1} ; \lambda_{1}, \ldots, \lambda_{n}\right)$, we have

$$
N_{\pi}\left(x_{1}, \ldots, x_{n-1} ; \lambda_{1}, \ldots, \lambda_{n}\right)=\sum_{k=1}^{n} N_{\pi, k}(0),
$$

and so

$$
\sum_{\pi} N_{\pi}\left(x_{1}, \ldots, x_{n-1} ; \lambda_{1}, \ldots, \lambda_{n}\right)=\sum_{k=1}^{n} N_{k}(0)=n ! .
$$

Proof of the theorem : 
Call $\left(x_{1}, x_{2}, \ldots, x_{n-1}\right)$ the rigid points and $x_{n}$ the free point. Define a finite set $B=B\left(x_{1}, \ldots, x_{n-1} ; \lambda_{1}, \ldots, \lambda_{n}\right)$ of "bad" points as follows : for a rigid point $x_{i}$ and a subset $\Sigma \subseteq\{1, \ldots n\}$, consider the point $x_{i}+\sum_{k \in \Sigma} \lambda_{k} ; B$ is comprised of all such points, as $i$ ranges over $1 \leq i \leq n-1$ and $\Sigma$ ranges over the subsets of $\{1, \ldots, n\}^{3}$. The theorem will be shown to apply to all $y \notin B$.

We prove the theorem by induction on $n$. Start with $n=1$. There are no rigid points, so $B=B\left(; \lambda_{1}\right)$ is empty. For every $y$ there is exactly one choice for the free point, namely $y-\lambda_{1}$, such that the rod $\lambda_{1}$, which must necessarily be assigned to the free point, departs at $y$.

To propagate the induction, suppose the theorem has been proved for all configurations of $m-1$ rigid points and $m$ positive rod lengths for all $1 \leq m \leq n-1$. We will now prove the theorem for $\left(x_{1}, \ldots, x_{n-1} ; \lambda_{1}, \ldots, \lambda_{n}\right)$.

Let $y \notin B$. We first claim that it suffices to consider $y>x_{n-1}$. Indeed, if $y<x_{1}$ then $y_{\pi, k}=y$ iff we set $x_{n}=y-\lambda_{k}$ and the permutation $\pi$ assigns $\operatorname{rod} \lambda_{k}$ to $x_{n}$ and the other $n-1$ rods to the rigid points in some order, and there are exactly $(n-1)$ ! ways of doing this. Also, if $x_{m}<y<x_{m+1}$ for some $1 \leq m \leq n-2$, for each of the $(n-1)(n-2) \ldots(m+1)$ ways of assigning $n-m-1$ of the rods other than $\lambda_{k}$ to the rigid points $x_{m+1} \leq \ldots \leq x_{n-1}$, by inductive hypothesis there are exactly $m$ ! choices for the location of the free point and assignments of the remaining rods to the remaining points (the free point and the remaining rigid points) which result in $\operatorname{rod} \lambda_{k}$ departing at time $y^{4}$, so the claim is proved.

Consider now $y \notin B$ with $y>x_{n-1}$. For $l \neq k$, consider the configuration $\left(x_{1}, \ldots, x_{n-2} ; \lambda_{1}, \ldots, \lambda_{l-1}, \lambda_{l+1}, \ldots, \lambda_{n}\right)$. By inductive hypothesis there are exactly $(n-2)$ ! choices for the location of the free point, which we will denote $x_{F}$, and for the assignment of rods other than $\lambda_{l}$ to $x_{F}$ and the points $x_{1} \leq \ldots \leq x_{n-2}$, which assignment we will denote by $\eta$, such that $\operatorname{rod} \lambda_{k}$ departs at $y$.

For such $x_{F}$ and $\eta$, suppose the rod $\lambda_{k}$ was assigned to one of the rigid points $x_{i}, 1 \leq i \leq n-2$, under $\eta$. Then the busy cycle of the FIFO queue that was in effect at time $x_{i}$ continues to be in effect throughout the interval $\left(x_{i}, y\right)$, which includes $x_{n-1}$. Thus, in the original problem $\left(x_{1}, \ldots, x_{n-1} ; \lambda_{1}, \ldots, \lambda_{n}\right)$,

\footnotetext{
${ }^{3}$ While it is not really necessary to include $\Sigma=\emptyset$ in the definition of $B$, it is convenient to not allow $y$ in the theorem to be one of the rigid points.

${ }^{4}$ The bad set for the configuration of the remaining rigid points and the remaining rod lengths is contained in the original bad set $B$.
} 
choosing $x_{n}=x_{F}$ and assigning rods to points according to $\pi$ which extends $\eta$ by assigning rod $\lambda_{l}$ to $x_{n-1}$, will also have rod $\lambda_{k}$ departing at time $y$.

Next, for such $x_{F}$ and $\eta$, suppose the rod $\lambda_{k}$ was assigned to $x_{F}$ and had to wait before beginning service. Then there must be some $x_{i}, 1 \leq i \leq n-2$, which initiated the busy cycle in which the $\operatorname{rod} \lambda_{k}$, which arrives at $x_{F}$, begins waiting. This would then mean that $y \in B$, which we have explicitly disallowed. Thus this case cannot occur.

It remains to consider those choices of $x_{F}$ and $\eta$ for which the rod $\lambda_{k}$ was assigned to $x_{F}$ and began to be served immediately on arrival at time $x_{F}$. This means $x_{F}=y-\lambda_{k}$.

In such a case, suppose $x_{F}<x_{n-1}$. Then $x_{n-1}$ arrives during the service period $\left(x_{F}, x_{F}+\lambda_{k}=y\right)$ of $\operatorname{rod} \lambda_{k}$. Once again, in the original problem $\left(x_{1}, \ldots, x_{n-1} ; \lambda_{1}, \ldots, \lambda_{n}\right)$, choosing $x_{n}=x_{F}$ and assigning rods to points according to $\pi$ which extends $\eta$ by assigning $\operatorname{rod} \lambda_{l}$ to $x_{n-1}$, will have $\operatorname{rod} \lambda_{k}$ departing at time $y$.

Next, suppose $x_{F}>x_{n-1}$. Let $z$ denote the end of the most recent busy cycle before $x_{F}$. If $\left(z \vee x_{n-1}\right)+\lambda_{l}<x_{F}$ the choice, in the original problem, of $x_{n}=x_{F}$ and the assignment of rods to points by $\pi$ which extends $\eta$ by assigning rod $\lambda_{l}$ to $x_{n-1}$ will again have rod $\lambda_{k}$ departing at time $y$, because the service of rod $\lambda_{l}$ will begin at $z \vee x_{n-1}$ and be completed before $x_{F}$. Note that this case can occur only if $x_{F}$ and $\eta$ are such that

$$
y>\left(z \vee x_{n-1}\right)+\lambda_{l}+\lambda_{k}
$$

We are finally left with one case for $x_{F}$ and $\eta$ : the rod $\lambda_{k}$ was assigned to $x_{F}$ under $\eta$, began service immediately on arrival, and

$$
\left(z \vee x_{n-1}\right)<x_{F}<\left(z \vee x_{n-1}\right)+\lambda_{l}
$$

where $z$ denotes the end of the most recent busy cycle before $x_{F}$. Note that $x_{F}=y-\lambda_{k}$ in this case. Also note that this case can occur only if $x_{F}$ and $\eta$ are such that

$$
y<\left(z \vee x_{n-1}\right)+\lambda_{l}+\lambda_{k}
$$

Thus, for any $y$ either this case or the preceding case occurs, but not both.

In this case, in the original problem, we assign the rods to points according to $\pi$ which equals $\eta$ for all rods other than $\lambda_{k}$ and $\lambda_{l}$, and assigns $\lambda_{k}$ to $x_{n-1}$ 
and $\lambda_{l}$ to the free point. Note that

$$
\begin{aligned}
\left(z \vee x_{n-1}\right)+\lambda_{k} & <y \text { and } \\
y-\left[\left(z \vee x_{n-1}\right)+\lambda_{k}\right] & <\lambda_{l} .
\end{aligned}
$$

We place the free point, in the original problem, at the point $x_{n}<x_{n-1}$ such that

$$
I\left(x_{n}, x_{n-1}\right)+y-\left[\left(z \vee x_{n-1}\right)+\lambda_{k}\right]=\lambda_{l},
$$

where $I\left(x_{n}, x_{n-1}\right)$ denotes the total idle time of the FIFO queue associated to $x_{F}$ and $\eta$ over the interval $\left(x_{n}, x_{n-1}\right)$. With this choice of free point $x_{n}$ and assignment $\pi$ in the original problem, the rod $\lambda_{k}$ departs at $y$.

We have now shown that, in the original problem, for each $l \neq k$ there are at least $(n-2)$ ! choices for $x_{n}$ and an assignment $\pi$ of rods to points such that rod $\lambda_{k}$ departs at time $y$ and either (1-l) $x_{n-1}$ is assigned rod $\lambda_{l}$ or (2-1) $x_{n-1}$ is assigned rod $\lambda_{k}$ and $x_{n}$ is assigned rod $\lambda_{l}$. We will now prove that for each $l \neq k$ there are exactly $(n-2)$ ! choices for $x_{n}$ and an assignment $\pi$ of rods to points such that "(1-l) or (2-l) and rod $\lambda_{k}$ departs at time $y$ " holds. Since the assignments satisfying "(1-l) or (2-l)" are disjoint as $l$ ranges over $l \neq k$ and cover all possible assignments, this would complete the inductive step and the proof of the theorem.

Fix $l \neq k$ and a choice of $x_{n}$ and an assignment $\pi$ of rods to points such that "(1-1) or (2-1) and rod $\lambda_{k}$ departs at time $y$ " holds. We show that each such $x_{n}$ and $\pi$ corresponds to a distinct choice of free point, which we denote $x_{F}$, and assignment, which we denote $\eta$, in the configuration $\left(x_{1}, \ldots, x_{n-2} ; \lambda_{1}, \ldots, \lambda_{l-1}, \lambda_{l+1}, \ldots, \lambda_{n}\right)$, which we call the reduced configuration, such that rod $\lambda_{k}$ departs at time $y$ in the reduced configuration. By the inductive hypothesis it would follow that there at most $(n-2)$ ! such assignments, which would complete the proof of the theorem.

Suppose $x_{n}$ and $\pi$ are such that (1-l) holds and rod $\lambda_{k}$ departs at time $y$ in the original configuration. If $\pi$ assigns $\operatorname{rod} \lambda_{k}$ to one of the original rigid points $x_{i}, 1 \leq i \leq n-2$, the busy cycle containing $x_{i}$ must last through the interval $\left(x_{i}, y\right)$, which contains $x_{n-1}$, so in the reduced configuration we could take $x_{F}=x_{n}$ and $\eta$ to be the restriction of $\pi$ that ignores the assignment of $\operatorname{rod} \lambda_{l}$ to $x_{n-1}$, and we would then have rod $\lambda_{k}$ departing at time $y$ in the reduced configuration.

Suppose $x_{n}$ and $\pi$ are such that (1-1) holds and rod $\lambda_{k}$ departs at time $y$ in the original configuration. If $\pi$ assigns $\operatorname{rod} \lambda_{k}$ to $x_{n}$, it must be the 
case that rod $\lambda_{k}$ does not have to wait before beginning service. Indeed, if it did have to wait before beginning service, this would have been because it arrived in some busy cycle which was initiated by some one of the rigid points $x_{i}, 1 \leq i \leq n-1$, but that would then mean that $y \in B$, which we have explicitly disallowed. Thus, we have $x_{n}=y-\lambda_{k}$. This then means that in the reduced configuration we could take $x_{F}=x_{n}$ and $\eta$ to be the restriction of $\pi$ that ignores the assignment of $\operatorname{rod} \lambda_{l}$ to $x_{n-1}$, and we would then have rod $\lambda_{k}$ departing at time $y$ in the reduced configuration.

Note that there are two ways in which the case just discussed could have occured in the original configuration : either (a) $x_{n}<x_{n-1}$ or (b) $x_{n}>x_{n-1}$. In the former case our choice of $x_{F}$ and $\eta$ results in $x_{F}<x_{n-1}$. In the latter case, the fact that the rod $\lambda_{k}$, which was assigned to $x_{n}$, does not have to wait before beginning service means that

$$
x_{n}>x_{n-1}+\lambda_{l}
$$

and also

$$
x_{n}>z+\lambda_{l}
$$

where $z$ denotes the departure time of the rod assigned to $x_{n-2}$. Recall that $x_{n}=y-\lambda_{k}$. Thus, this case can only occur if we had

$$
y>\left(z \vee x_{n-1}\right)+\lambda_{k}+\lambda_{l}
$$

Also note that $z$ can be computed based purely on the restriction of the assignment $\pi$ (or equivalently $\eta$ ) to the rigid points $x_{i}, 1 \leq i \leq n-2$.

Finally, suppose $x_{n}$ and $\pi$ are such that (2-l) holds and rod $\lambda_{k}$ departs at time $y$ in the original configuration. Then, because rod $\lambda_{k}$ begins service at $y-\lambda_{k}$ and $y \notin B$, it must be the case that $x_{n-1}$ lies in a busy cycle initiated by $x_{n}$. In the reduced configuration consider the assignment $\eta$ that assigns rods to the rigid points $x_{i}, 1 \leq i \leq n-2$, exactly as $\pi$ does, and assigns rod $\lambda_{k}$ to the free point $x_{F}=y-\lambda_{k}$. We then have rod $\lambda_{k}$ departing at time $y$ in the reduced configuration.

The choice of $x_{F}$ and $\eta$ we have created from $x_{n}$ and $\pi$ in this case is identical to that in sub-case (b) of the preceding case. However, recall that this case could only have occured if $x_{n-1}$ lies in the busy cycle initiated by $x_{n}$. Let $z$ denote the departure time of the rod assigned to $x_{n-2}$ when one considers only the rods assigned to the rigid points $x_{i}, 1 \leq i \leq n-2$, 
according to the restriction of the assignment $\pi$ (or equivalently $\eta$ ). The work remaining in the system at time $x_{n-1}$ in the original configuration is then strictly less than $\left(z \vee x_{n-1}\right)-x_{n-1}+\lambda_{l}$. It follows that

$$
y-\lambda_{k}-x_{n-1}<\left(z \vee x_{n-1}\right)-x_{n-1}+\lambda_{l},
$$

which is to say that

$$
y<\left(z \vee x_{n-1}\right)+\lambda_{k}+\lambda_{l} .
$$

Thus, this case cannot occur for the same values of $y$ for which sub-case (b) of the preceding case occurs. This completes the proof of the theorem.

\section{References}

[1] S. Asmussen, Applied Probability and Queues, John Wiley and Sons, 1987.

[2] A. Rybko and S. Shlosman, Poisson hypothesis for information networks. I : Domain of validity, Math Arxiv, paper No. math PR/0406110. 